This is a preprint of

Luciano Floridi, (1996) "Brave.Net.World: the Internet as a disinformation superhighway?", The Electronic Library, Vol. 14 Issue: 6, pp.509514, https://doi.org/10.1108/eb045517

Permission to make digital or hard copies of all or part of this work for personal or classroom use is granted without fee provided that copies are not made or distributed for profit or commercial advantage and that copies bear this notice and the full citation on the first page. To copy otherwise, or republish, to post on servers or to redistribute to lists, requires prior specific permission and/or a fee.

It is a publisher's requirement to display the following notice:

The documents distributed by this server have been provided by the contributing authors as a means to ensure timely dissemination of scholarly and technical work on a noncommercial basis. Copyright and all rights therein are maintained by the authors or by other copyright holders, notwithstanding that they have offered their works here electronically. It is understood that all persons copying this information will adhere to the terms and constraints invoked by each author's copyright. These works may not be reposted without the explicit permission of the copyright holder.

In the case of Springer, it is the publisher's requirement that the following note be added:

"An author may self-archive an author-created version of his/her article on his/her own website and his/her institution's repository, including his/her final version; however he/she may not use the publisher's PDF version which is posted on www.springerlink.com. Furthermore, the author may only post his/her version provided acknowledgement is given to the original source of publication and a link is inserted to the published article on Springer's website. The link must be accompanied by the following text: "The original publication is available at www.springerlink.com." 


\section{Brave.Net.World: The Internet as a Disinformation Superhighway?}

Luciano Floridi ${ }^{1,2}$

${ }^{1}$ Oxford Internet Institute, University of Oxford, 1 St Giles, Oxford, OX1 3JS, United Kingdom; ${ }^{2}$ The Alan Turing Institute, 96 Euston Road, London, NW1 2DB, United Kingdom.

Miranda: "Oh brave new world that has such people in it". Shakespeare, The Tempest (ca. 1611)

"They will grow up with what the psychologists used to call an 'instinctive' hatred of books and flowers. Reflexes unalterably conditioned. They'll be safe from books and botany all their lives" Huxley, Brave New World (1932)

"Die breite Masse eines Volkes [...] einer grossen Lüge leichter zum Opfer fällt als einer kleinen.

The broad mass of a nation [...] will more easily fall victim to a big lie than to a small one." Adolf Hitler, Mein Kampf(1925)

\section{Overture: the problem}

Nobody could seriously doubt that the unidirectional mass media can be very powerful instruments of disinformation. History has already witnessed too many horrible events to allow ourselves the luxury of such futile speculation any longer.[1] What we might do instead is to turn our attention to the brave new world of the Internet, and ask whether the problem of disinformation might soon afflict the new interactive media as well.

Suppose that in years to come there will still be a significant dissimilarity between passive (one way, or simply W) and interactive (two way, or simply WW) media. The management of information online is going to affect many aspects of our life increasingly commonly[2], and the following three questions will become crucial:

1) will the Internet too become a powerful means of potential disinformation? And if so, 
2) will disinformation engendered via the Internet differ from other forms of disinformation engendered via paper and broadcasting media? And finally,

3) if the Internet could become a powerful means of disinformation, is there anything that can be done to avoid this particular problem or to solve it?

It is my impression that each of these questions can be answered in the positive. This paper's task is to attempt to explain why and in what sense.

\section{The starting point: from questions to assumptions}

The three questions just stated presuppose that:

i) the Internet is (going to be) a new mass medium; and

ii) because of (i) the Internet cannot avoid the problem of disinformation.

We cannot endorse (i) without a proviso which will, I hope, acquire its full significance in section 6: nowadays the Internet is really an instrument of information and communication only within a socio-cultural elite of a few million people and hence it is better described as a group medium rather than a mass medium[3]. And we cannot accept (ii) unless we answer two further questions first:

4) what do we mean by the problem of disinformation? And

5) is any mass medium - and hence the Internet as well - bound to face it?

Let us deal with question (4) first.

\section{One step back: three forms of disinformation}

Disinformation arises whenever the process of information is defective. This can happen because of:

a) a lack of objectivity, as in the case of propaganda [4];

b) a lack of completeness, as in a case of damnatio memoriae;

c) a lack of pluralism, as in the case of censorship [5].

Each type can be combined with the other two in more complex and efficient forms of disinformation, but this is irrelevant here. More to the point is to note that, contrary to what the examples seem to suggest, each form of disinformation need not necessarily be intentional. I shall come back to this qualification in a moment. But first, let us concentrate on question (5). 


\section{Another step back: disinformation as an endogenous problem}

Past analyses of W-media, like newspapers, radio or television, cannot merely be extended to the new world of online communication and WW-media. We cannot exclude a priori the possibility that technical differences between the media may give rise to differences in the nature of the disinformation they make possible. More explicitly, disinformation via TV may be a different phenomenon from disinformation achieved via the Internet, just as advertising via the two media differs. We shall see that this is actually the case. So we had better keep the two separate and avoid confusion. Having granted this point, however, I would contend that we are still entitled to use the same conceptual framework, and hence to apply the concept of disinformation to the Internet as well, for the following reason. The management of information can be affected by three types of problems (whether they are ethical, legal or just practical does not matter here):

1) problems arising from what can be done to information throughout its lifecycle (especially but not only creation, storage, retrieval, updating). We find here problems arising from possible loss or damage due to software virus, fire, chemical agents, misplacement, theft or the ageing of a particular technology, from the lack of physical or magnetic space, from the necessity of out-sourcing, from spying, hacking or terrorist attacks, and so forth;

2) problems arising from what can be done with information. Examples here can be as disparate as blackmailing, insider trading, infoglut or plagiarism; and

3) problems concerning both the life-cycle and the use of information. Two typical cases are those of pornography and of privacy of communication.

Disinformation is caused by some form of mishandling of information, belongs to the third group and is endogenous to any information management system (IMS), from the manuscript tradition to the card index of a library, from the publication of a scholarly journal to the broadcasting of a popular radio program. Now, all media of any kind are IMS, and since we have assumed that the Internet is a medium, the consequences are that:

a) the Internet, today represented mainly by the WWW, cannot avoid the problem of disinformation, not just because it is comparable to other W- or WW-media - this is superficial - but because it is another particular instance of a medium, any medium is an IMS, and any IMS faces disinformation for the very reason that IMS constantly run the risk of mishandling their documents; 
b) since disinformation is an endogenous problem of any IMS, in the case of the Internet too it may arise at any time; it cannot merely be evaded but must be confronted explicitly.

\section{One side-step: involuntary disinformation}

Given the context of the human management of information, no stage in the epistemic process - from the initial creation of data to the final use of the corresponding information - is thoroughly transparent. This implies that a certain degree of involuntary disinformation (lack of objectivity, completeness and pluralism) can occur in any IMS taken into account. With a difference, which represents a first answer to question 2 . With passively-consumed mass media the problem is mainly one of unpremeditated creation of disinformation. Whenever information passes from the sender to the receiver it runs the risk of being corrupted or mutilated. One can think of a medieval copier's oversight, or of the limited space given by a TV programme to a particular event. In the case of the Internet, the increasing facility and speed with which mono- or multimedia documents can be created, manipulated, reproduced and spread makes the problem of involuntary diffusion of disinformation more acute. A nice example is provided by the message concerning an alleged virus, called "Good Times", that keeps on appearing over and over again in many email lists. It's a hoax, but overconcerned and unaware users keep on forwarding it so easily that it has been impossible to restrain the diffusion of this particular disinformation for more than two years now. [6] It is for this reason that more and more often email lists which are disinformation-sensitive have disclaimers automatically included in their messages, specifying for example, that "NEW-LIST announcements are edited from information provided by the original submitter. We do NOT verify the technical accuracy nor any claims made in the announcements nor do we necessarily agree with them. We do not warranty or guarantee any services which might be announced - use at your own risk. [...]".[7]

\section{Back to the starting point: disinformation via the Internet now}

Having dealt with unpremeditated forms of disinformation, let us concentrate now on voluntary disinformation. Our first question was whether the Internet too will become a powerful means of potential disinformation. Two views should be distinguished. In spite of some clear cases of disinformation[8], at the moment there seem to be no reasons to be worried. The Internet has not yet provided us with a powerful means of 
disinformation, especially if we interpret the adjective "powerful" by contrasting it to what the unidirectional media enable us to do already. Things may stand rather differently when we consider what may happen during the first decade of the next millenium.

A system of information management and communication can generate disinformation with increasing efficiency the more the following three conditions are satisfied:

a.1) if there occurs a dichotomy between the sender, who possesses and provides the information, and the receiver, who lacks it. Note that, given this gap, disinformation is easier the more authoritative[9] and influential its source and the more naive the population it targets;

a.2) the easier it is, on the side of the sender, to censor (that is to cast out and suppress) other sources of de-disinformation (denials, corrigenda and addenda), as quietly as possible, so that the very process of censorship does not become a matter of information itself;

a.3) the more difficult it is, on the side of the receiver, to control the level of objectivity, completeness and pluralism of the information.

The better these three conditions of ignorance, coercion, and impotence are fulfilled, the more powerful a mass medium can be in terms of producing disinformation. Now, although in different degrees, there have been plenty of cases in which the unidirectional mass media have been able to satisfy all three conditions rather well. This does not yet hold good for the Internet, for three corresponding reasons:

b.1) there exists, at the moment, a lower degree of imbalance between the providers and the users of information.

This is owing to two factors. One is contingent: the Internet is actually being used by a socio-cultural elite whose members would find it more difficult to disinform one another because, to a large extent, this is also the educated elite that can keep the life and flow of information under control. The other is necessary: the Internet is interactive and, when compared to other mass media, much cheaper. These two factors have the result that, contrary to what happens in the case of the passive media, the relation between provider and user is interchangeable and can be direct: every user can become a provider of information (a BBS or a WWW page is sufficient), and the transaction between provider and user of information does not necessarily require an intermediary. If now we put aside the increasing need to delegate the certification of the quality of the information 
exchanged to organizations of various types (libraries, universities, publishers, public institutions, international organizations, private companies and so forth), the growth of a plurality of sources of information enhances mass production of information, which in turn should increase a correct (i.e. not disinformative) use of it. In short, it is certainly true that the chances of successfully spreading disinformation decrease as the number of provusers (providers and users) of information increases. The unfortunate thing is that this is only half the story, and we shall see shortly that matters are a bit more complex.

b.2) difficulty of censorship

The wider the plurality of information, the smaller the risks of disinformation. A necessary condition for plurality of information is the occurrence of a variety of providers. Now the variety of providers is opposed, mainly for economical reasons, by monopolistic groups (which in turn are opposed, mainly for political reasons, by antitrust legislations), while it is promoted by the increase in the number of different types of mass media. We have assumed that the Internet is at least a new group medium. This means that its growth, alongside the other passive media, has potentially increased the plurality of information, and thus reduced the risk of disinformation.

\section{b.3) Ease of control}

Interactivity also means an opening-up of information system, which strives to become constantly available and easily accessible to the largest possible number of people in affordable ways. Of course this implies more serious difficulties for those who wish to propagate disinformation efficiently.

To summarise: since the Internet is presently an interactive group medium used by a restricted elite which, to a large extent, is capable of controlling the world of information, it is also a much less efficient instrument of disinformation than any other unidirectional mass medium.

\section{A step into the future: when massive disinformation will be possible via the}

\section{Internet}

Unfortunately, things may easily become more problematic in the future, for reasons connected to two variables - the number of provusers and the physical integration of the various mass media into a unique digital instrument - and a constant factor, represented by the interactive technology. The three reasons can be presented schematically thus: 
c.1) there will be the return of a certain gap between information providers and information users. This is due to the increase in the number of provusers, which is already causing a new fragmentation of the electronic agora. There is a "physiological limit" to the globality of interactive information, and the Internet reached it in 1994. To give an example, nobody today would be able to control, or even just take part in, the thousands of e-lists active all over the world. Now the fragmentation of the society of provusers into a mosaic of interest groups implies the reappearance on a horizontal scale, as it were, of the dichotomy between provider and user of information, and thus

- a new increase in the possibilities for disinformation;

- an increase in the number of attempts to effect disinformation via the network. The more people are online, the more likely it becomes that some of them will have the desire and the technical capacities (mass interaction) to disinform on an international scale with some success;

- disinformation via the network to become more interesting. Very soon, the number of people on the Internet will be high enough to justify the effort needed to disinform them, exactly in the same way that the growth of the number of provusers will increasingly attract the attention of advertising companies[10];

c.2) digital integration[11]

Insofar as the process of integration and digital harmonisation of the various mass media will succeed in pulling down the technological barriers still existing between them, it will also weaken the advantages arising from a plurality of types of mass media and hence of information. Thus it will increase the risks connected with the emergence of monopolies, which will in turn potentially facilitate the spreading of disinformation;

\section{c.3) the development of interactivity}

Interactive mass media could give rise to self-disinformation, i.e. a new form of personal disinformation which has so far remained largely a potential problem. . This is a second way in which disinformation engendered via WW-media differs from disinformation engendered via W-media (see question 2). Today, we are subject to a broad range of information, whether we like it or not. We usually absorb, in a passive way, quite extensive blocks of ready-to-consume information: a whole book, a newspaper, a periodical, an entire radio programme, the whole news on TV, a cinema season and so forth. And while the transformation of mass media into interactive media goes some way 
towards solving this problem, it also gives rise to new risks. Soon we may be able to personalise the information we need or wish to use, filtering and adjusting it to our tastes (information tailoring). And this means that the possibility will arise of listening only to the few sources we have tailored to our preferences, on a limited number of topics, and with a very specific angle of interpretation. But we shall run far greater risks of disinformation within such a comfortable data niche than those people who nowadays read only the newspaper of their own political party. Believing ourselves to be more and better informed, we would be increasingly conditioned by our idiosyncrasies.

\section{Finale allegro ma non troppo: three things that can be done}

The risks I have just analysed are only possibilities, which have to be balanced against the three points I made before in favour of a non-disinformative use of the Internet. For example, interactivity will always remain an easier and more democratic way of exercising forms of de-disinformation (at least as long as Harry's wishes are duly fulfilled[12]). But even if we limit our attention only to the problems and hence to our third question, I believe it will be possible to deal with them successfully if the following strategies are adopted.

\section{d.1) Quality certification}

The lack of balance between what is becoming available online and what can be examined by each provuser brings us to the problem of establishing services that can test and certify the integrity and quality of the information in question, and promote its plurality. I have already dwelt on this point in another article, and here I shall limit myself to repeating the conclusions reached in that context.[13] The problem of disinformation can be solved, at least partially, by relying on the interactive nature of the new medium, but this will be possible only if efficient instruments are implemented in order to monitor the information available and hence secure:

1) the reliability, integrity and plurality of the digital information[14];

2) constant access to the digital macrocosm for the largest number of people (universal right to the production and use of information); and

3) a constantly updated map of the digital universe of information available online.

Only from the perspective of these tasks can the similarity and importance of two recent phenomena be appreciated. The first is the renewed presence, in the field of information 
management, of academic institutions, which have started to work as vital centres of production and evaluation of online information, though not yet as quality controllers. The second is the appearance of commercial services to meet provusers' epistemic needs: search engines, on which paying sponsors advertise their products or display their logos, are increasingly commonly helping users who are lost in an ocean of information, and satisfying, at least to some extent, their need for a constantly updated map of the digital domain. What has not been done at all yet - and this is no small task - is to intervene in the nature of the documents available via the Internet in order to grant them the full status of information. Nowadays no newspaper, academic journal or news programme would include an explicit disclaimer of accountability for the information provided. The Internet has not yet reached this minimal level of reliability, as the CNN Web business service shows.[15]

\section{d.2) Anti-trust}

In the face of the process of integration and digital harmonisation of the various mass media, the best remedy is the promotion of plurality of information. Technology has already made its contribution. Today, practically anybody can become a craftsman of information on the Internet, at least in theory. But the real conflict will take place in the field of the great monopolies. And what has happened during 1995 in the United States shows that the first round has been lost by social and cultural interests and won by economic ones.

\section{d.3) Individual education}

Self-disinformation - ignoring information, or being accustomed to knowing things only in a distorted manner - is the easiest way humanity knows to survive the daily pressure of reality on the mind. For reasons that are mainly technological, our mass media have so far implicitly taken into account this negative anthropology and, to quote the famous passage from Luke 14,23, they have "forced us to enter" into the world of information. But when technological limits disappear, it will be up to us to overcome our mental inertia and embrace, deliberately, the Kantian and enlightened "sapere aude" that today is forced upon us from without.

Technology exacerbates the problems, but the fundamental questions remain human and social. It is a rather trivial conclusion, I am aware, but it will be political power and the way in which the new generations are educated that will determine 
whether the interactive mass media of the future will be better or worse than the present. There is nothing new under the sun, to stay with the biblical context.[16]

Notes

[1] Holocaust Internet Sites. You may also wish to have a look at the Institute for Historical Review). "Certainly the best-known and most controversial aspect of the IHR's work has been its treatment of the Holocaust issue." (from the home page). Simon Wiesenthal Center: "January 12, 1996, Wiesenthal Center Calls on Internet Providers To Adopt Voluntary Standard of Ethics. [...] In the wake of the growing number of organized hate groups espousing racism, antisemitism, violence and mayhem on the World Wide Web, the Simon Wiesenthal Center has called upon companies providing Internet hosting services to adopt voluntary acceptable-use guidelines that would terminate services to individuals or groups who incite mayhem or racist violence." from the home page.

[2] Among the many interesting Web sites covering the topic of ethical issues brought about by the third era of IT see http://www.cms.dmu.ac.uk/CCSR.

[3] CNN, The Internet: Will it last or fade into the past? - Jan. 14, 1996: "A recent survey found about 9.5 million people in the United States logging an average of six-and-a-half hours a week on-line. The figure is far lower than a study last summer that counted more than 24 million Internet users." For more details see the CommerceNet/Nielsen Internet Demographics Survey, based on Internet users in US and Canada. The Executive Summary is being distributed for free via the Internet on CommerceNet's and Nielsen Media Research's WWW servers. The Final Report is available for purchase from CommerceNet (phone: 415-617-8790; e-mail: survey@commerce.net) and Nielsen Media Research (phone: 813-738-3125; e-mail: interactive@nielsenmedia.com).

[4] Propaganda on the Internet? See http://www.kosone.com/people/ocrt/int_hate.htm

[5] Cf. the transcript of the FFE's (Feminists For Free Expression) Censorship in Cyberspace forum.

[6] See for example 
http://nethelp.tamu.edu/ swood/GoodTimes-HoaxFAQ.html

http://www.tcp.co.uk/tcp/good.times.html, http://www.informatik.uni-trier.de/ bern/GoodTimes-Hoax/.

On the risk of enhancing disinformation by repetitive false alarms (desensitization, like in the famous fable about "crying wolf" ) see Rapid dissemination of half-truths, lies, and disinformation published in _The Risks Digest_ 11.41 Monday 8 April 1991 by J. E. Oberg.

[7] This disclaimer comes from the philosophical list Philos-L.

[8] For a collection of links on the problem of disinformation via the Internet and the quality of Web information see Robert Stepno's and Paul Jones' Disinformation Pages.

[9] For an entertaining selection of involuntary authoritative examples of disinformation, see Great Quotes from Great Skeptics. All quotations come from The Experts Speak: The Definitive Compendium of Authoritative Misinformation. Here is one from a prestigious colleague: "[W] hen the Paris Exhibition closes electric light will close with it and no more be heard of". Erasmus Wilson (1878) Professor at Oxford University.

[10] Federal Trade Commission Home Page, see the article on "Rule Banning Deceptive, Abusive Telemarketing" and "Online Scams: Road Hazards on the Information Superhighway" from which the following quote is taken: "Cyberspace has become the new frontier for scam artists. The scams aren't new, just the medium. Fraudulent sellers use computer services to promote familiar schemes such as bogus stock offerings, hightech investment opportunities, and credit-repair services. Treat all ads or would-be ads with skepticism and never make an investment or health-related purchase decision based solely on information obtained from a single source in any medium-- print, broadcast, or online."

[11] I do not say "unification" because I believe no complete digital unification of mass media will be achieved in the future, but I must leave the discussion of this point to another article. 
[12] The following passage provides a bit of information I suppose the CNN people did not mean to make available. The line on Harry's wishes does not appear on the screen but it is downloaded by Netscape and appears in the file:

"CNN - New survey finds Internet use is surging - Jan. 13, 1996

http://www.cnn.com/TECH/9601/internet_use/index.html [...]

$<!--------D O N O T$ REMOVE FEEDBACK - Harry wants it on all stories------------>

$<$ BR CLEAR $=$ ALL $><$ HR WIDTH $=40 \%>$

$<\mathrm{H} 3>$ Feedback $</ \mathrm{H} 3>$

$<\mathrm{ul}>$

$<\mathrm{li}><\mathrm{A} \quad \mathrm{HREF}=$ "/feedback/index.html" $>$ Send us $</$ a $>$ your comments - $<\mathrm{A}$ $\mathrm{HREF}=$ " / feedback/comments.html" $>$ Selected responses $</ \mathrm{A}>$ are posted daily.

$</$ ul $>$

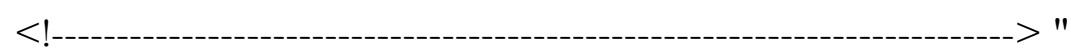

Obviously, the indication not to remove the feedback was not meant to be selfreferential.

[13] See The Internet: which future for organised knowledge, Frankenstein or Pygmalion?.

[14] For examples of mass media monitoring organizations see:

FAIR -- Fairness \& Accuracy In Reporting, "is the national (USA) media watch group offering well-documented criticism in an effort to correct media bias and imbalance. [...] FAIR seeks to invigorate the First Amendment by advocating for greater media pluralism and the inclusion of public interest voices in national debates." from the home page.

Media Watchdog " is a collection of online media watch resources, including specific media criticism articles and information about media watch groups. The emphasis is on critiquing the accuracy and exposing the biases of the mainstream media." from the home page.

EIC "The Environmental Information Center is an organization founded in November 1994 to combat environmental misinformation and help strengthen grassroots support for environmental protection." from the home page. 
[15] This is the disclaimer appearing at the end of the business page of the CNN Internet service: "In addition to the terms and conditions of the CNN Interactive Subscriber Agreement which govern the use of CNN Interactive, please be advised that neither, Cable News Network, Inc. ("CNN") nor its affiliates, information providers or content partners shall be liable regardless of the cause or duration, for any errors, inaccuracies, omissions, or other defects in, or untimeliness or unauthenticity of, the information contained within CNN Interactive, or for any delay or interruption in the transmission thereof to the user, or for any claims or losses arising therefrom or occasioned thereby. None of the foregoing parties shall be liable for any third-party claims or losses of any nature, including, but not limited to, lost profits, punitive or consequential damages. Prior to the execution of a stock trade, you are advised to consult with your broker or other financial representative to verify pricing information. $\mathrm{CNN}$, its affiliates, information providers or content partners shall have no liability for investment decisions based on the information provided. Neither, CNN, nor its affiliates, information providers or content partners warrant or guarantee the timeliness, sequence, accuracy or completeness of this information. Additionally, there are no warranties as to the results obtained from the use of the information." The first part seems fair, but what about accuracy and completeness?

[16] This article is a modified version of a paper I gave to the conference Philosophy \& Informatics - First Italian Conference on the use of ICT in philosophical disciplines, promoted by the Italian Philosophical Association (University of Rome "La Sapienza", 23-24 November, 1995). I wish to thank Bruno Ballardini, Carla Bazzanella, and Mario Jori for their comments and suggestions on previous drafts. 